JOURNAL OF THE

AMERICAN MATHEMATICAL SOCIETY

Volume 16, Number 1, Pages 57-67

S 0894-0347(02)00402-2

Article electronically published on July 29, 2002

\title{
FAMILIES OF RATIONALLY CONNECTED VARIETIES
}

\author{
TOM GRABER, JOE HARRIS, AND JASON STARR
}

\section{INTRODUCTION}

1.1. Statement of results. We will work throughout over the complex numbers, so that the results here apply over any algebraically closed field of characteristic 0 .

Recall that a proper variety $X$ is said to be rationally connected if two general points $p, q \in X$ are contained in the image of a map $g: \mathbb{P}^{1} \rightarrow X$. This is clearly a birationally invariant property. When $X$ is smooth, this turns out to be equivalent to the a priori weaker condition that two general points can be joined by a chain of rational curves, and also to the a priori stronger condition that for any finite subset $\Gamma \subset X$ there is a map $g: \mathbb{P}^{1} \rightarrow X$ whose image contains $\Gamma$ and such that $g^{*} T_{X}$ is an ample bundle.

Rationally connected varieties form an important class of varieties. In dimensions 1 and 2 rational connectivity coincides with rationality, but the two notions diverge in higher dimensions and in virtually every respect the class of rationally connected varieties is better behaved. For example, the condition of rational connectivity is both open and closed in smooth proper families; there are geometric criteria for rational connectivity (e.g., any smooth projective variety with negative canonical bundle is rationally connected, so we know in particular that a smooth hypersurface $X \subset \mathbb{P}^{n}$ of degree $d$ will be rationally connected if and only if $\left.d \leq n\right)$, and there are, at least conjecturally, numerical criteria for rational connectivity (see Conjecture 1.6 below). In this paper we will prove a conjecture of Kollár, Miyaoka and Mori that represents one more basic property of rational connectivity (also one not shared by rationality): if $X \rightarrow Y$ is a morphism with rationally connected image and fibers, then the domain $X$ is rationally connected as well. This will be a corollary of our main theorem:

Theorem 1.1. Let $f: X \rightarrow B$ be a proper morphism of complex varieties with $B$ a smooth curve. If the general fiber of $f$ is rationally connected, then $f$ has a section.

Since this is really a statement about the birational equivalence class of the morphism $f$, we can restate it in the equivalent form

Theorem 1.2. If $K$ is the function field of a curve over $\mathbb{C}$, then any rationally connected variety $X$ defined over $K$ has a $K$-rational point.

Received by the editors September 6, 2001 and, in revised form, May 3, 2002.

2000 Mathematics Subject Classification. Primary 14M20, 14D05.

The first author was partially supported by an NSF Postdoctoral Fellowship.

The second author was partially supported by NSF grant DMS9900025.

The third author was partially supported by a Sloan Dissertation Fellowship. 
In this form, the theorem directly generalizes Tsen's theorem, which is exactly this statement for $X$ a smooth hypersurface of degree $d \leq n$ in projective space $\mathbb{P}^{n}$ (or more generally a smooth complete intersection in projective space with negative canonical bundle; cf. [K] Theorem IV.6.5]). It would be interesting to know if in fact rationally connected varieties over other $C_{1}$ fields necessarily have rational points.

As we indicated, one basic corollary of our main theorem is

Corollary 1.3. Let $f: X \rightarrow Y$ be any dominant morphism of complex varieties. If $Y$ and the general fiber of $f$ are rationally connected, then $X$ is rationally connected.

Proof. Since we work over $\mathbb{C}$, we can assume that $X$ and $Y$ are smooth projective varieties. Let $p$ and $q$ be general points of $X$. We can find a map $g: \mathbb{P}^{1} \rightarrow Y$ whose image contains $f(p)$ and $f(q)$; let $X^{\prime}=\mathbb{P}^{1} \times_{Y} X$ be the pullback of $f^{\prime}$ by $g$. By Theorem 1.1 there is a section $D$ of $X^{\prime}$ over $\mathbb{P}^{1}$. We can then connect $p$ to $q$ by a chain of rational curves in $X^{\prime}$ in three stages: connect $p$ to the point $D \cap X_{p}$ of intersection of $D$ with the fiber $X_{p}$ of $f$ through $p$ by a rational curve; connect $D \cap X_{p}$ to $D \cap X_{q}$ by $D$; and connect $D \cap X_{q}$ to $q$ by a rational curve in $X_{q}$.

There is a further corollary of Theorem 1.1 based on a construction of Campana and Kollár-Miyaoka-Mori: the maximal rationally connected fibration associated to a variety $X$ (see $[\mathrm{Ca}],[\mathrm{K}]$ or $[\mathrm{KMM}]$ ). Briefly, the maximal rationally connected fibration associates to a variety $X$ a (birational isomorphism class of) variety $Z$ and a rational map $\phi: X \rightarrow Z$ with the properties that

- the fibers $X_{z}$ of $\phi$ are rationally connected; and conversely

- almost all the rational curves in $X$ lie in fibers of $\phi$ : for a very general point $z \in Z$ any rational curve in $X$ meeting $X_{z}$ lies in $X_{z}$.

The variety $Z$ and morphism $\phi$ are unique up to birational isomorphism and are called the mrc quotient and mrc fibration of $X$, respectively. They measure the failure of $X$ to be rationally connected: if $X$ is rationally connected, $Z$ is a point, while if $X$ is not uniruled, we have $Z=X$. As observed in [K] IV.5.6.3], we have the following corollary:

Corollary 1.4. Let $X$ be any variety and $\phi: X \rightarrow Z$ its maximal rationally connected fibration. Then $Z$ is not uniruled.

Proof. Suppose that $Z$ were uniruled, so that for a general point $z \in Z$ we could find a map $g: \mathbb{P}^{1} \rightarrow Z$ whose image contains $z$. By Corollary 1.3 , the pullback $X^{\prime}=\mathbb{P}^{1} \times_{Z} X$ of $\phi$ by $g$ will be rationally connected, which means that every point of the fiber $X_{z}$ will lie on a rational curve not contained in $X_{z}$, contradicting the second defining property of mrc fibrations.

There are conjectured numerical criteria for a variety $X$ to be either uniruled or rationally connected. They are

Conjecture 1.5. Let $X$ be a smooth projective variety. Then $X$ is uniruled if and only if $H^{0}\left(X, K_{X}^{m}\right)=0$ for all $m>0$.

Conjecture 1.6. Let $X$ be a smooth projective variety. Then $X$ is rationally connected if and only if $H^{0}\left(X,\left(\Omega_{X}^{1}\right)^{\otimes m}\right)=0$ for all $m>0$.

For each of these conjectures, the "only if" part is known and straightforward to prove; the "if" part represents a very difficult open problem (see for example [K, IV.1.12 and IV.3.8.1]). As another consequence of our main theorem, we have an implication: 


\section{Corollary 1.7. Conjecture 1.5 implies Conjecture 1.6}

Proof. This is proved in [K, IV.5.7], assuming Theorem 1.1; for completeness we remind the reader of the proof. Let $X$ be any smooth projective variety that is not rationally connected; assuming the statement of Conjecture 1.5, we want to show that $H^{0}\left(X,\left(\Omega_{X}^{1}\right)^{\otimes m}\right) \neq 0$ for some $m>0$. Let $\phi: X \rightarrow Z$ be the mrc fibration of $X$. By hypothesis $Z$ has dimension $n>0$, and by Corollary $1.4 Z$ is not uniruled. If we assume Conjecture 1.5, then we must have a nonzero section $\sigma \in H^{0}\left(Z, K_{Z}^{m}\right)$ for some $m>0$. But the line bundle $K_{Z}^{m}$ is a summand of the tensor power $\left(\Omega_{Z}^{1}\right)^{\otimes n m}$, so we can view $\sigma$ as a global section of that sheaf; pulling it back via $\phi$, we get a nonzero global section of $\left(\Omega_{X}^{1}\right)^{\otimes n m}$.

\section{Preliminary definitions and constructions}

We will be dealing with morphisms $\pi: X \rightarrow B$ satisfying a number of hypotheses, which we collect here for future reference. First of all, in proving Theorem 1.1, we are free to assume that $B$ is projective and that $X$ is smooth and projective by applying resolution of singularities and Chow's Lemma to $X$. For the bulk of this paper we will deal with the case $B \cong \mathbb{P}^{1}$; we will show in section 3.2 below both that the statement for $B \cong \mathbb{P}^{1}$ implies the full Theorem 1.1 and, as well, how to modify the argument that follows to apply to general $B$.

Hypothesis 2.1. $\pi: X \rightarrow B$ is a nonconstant morphism of smooth connected projective varieties over $\mathbb{C}$, with $B \cong \mathbb{P}^{1}$. For general $b \in B$, the fiber $X_{b}=\pi^{-1}(b)$ is rationally connected.

It is necessary to work with a class of maps of curves $g: C \rightarrow X$ more general than embeddings. We choose to work with Kontsevich's stable maps.

Definition 2.2. A pair $\left(f: C \rightarrow X,\left(p_{1}, \ldots, p_{n}\right)\right)$ of a morphism from a connected, complete, at-worst-nodal curve $C$ to $X$, and an ordered set of closed points, called marked points, of $C$ is a stable map if

(1) the marked points $p_{i}$ are all distinct,

(2) the marked points $p_{i}$ are contained in the smooth locus of $C$, and

(3) for every irreducible component $C_{j}$ of $C$ with arithmetic genus 0 on which $f$ is constant, there are at least three special points (nodes or marked points), and for every irreducible component $C_{j}$ of $C$ with arithmetic genus 1 on which $f$ is constant, there is at least one special point.

Given a numerical equivalence class $[D] \in N^{1}(X)$, the intersection number $f_{*}[C] .[D]$ is defined in the usual way. Thus $f_{*}[C]$ defines a class in $N_{1}(X)$. For a fixed nonnegative integer $g$, a fixed nonnegative integer $n$, and a class $\beta \in N_{1}(X)$, the Kontsevich moduli stack $\overline{\mathcal{M}}_{g, n}(X, \beta)$ is defined to be the Deligne-Mumford stack which parametrizes flat families of stable maps $\left(f: C \rightarrow X,\left(p_{1}, \ldots, p_{n}\right)\right)$ such that $C$ has arithmetic genus $g$ and the class $f_{*}[C]$ equals $\beta$. We denote by $\bar{M}_{g, n}(X, \beta)$ the coarse moduli space of $\overline{\mathcal{M}}_{g, n}(X, \beta)$. For definitions and results about Kontsevich stable maps, the reader is referred to [FP], [BM].

Now suppose we have a class $\beta \in N_{1}(X)$ having intersection number $d$ with a fiber of the map $\pi$. By [BM, Theorem 3.6] we have then a natural morphism

$$
\varphi: \bar{M}_{g, 0}(X, \beta) \rightarrow \bar{M}_{g, 0}(B, d)
$$

defined by composing a map $f: C \rightarrow X$ with $\pi$ and collapsing components of $C$ as necessary to make the composition $\pi f$ stable. 
Definition 2.3. Let $\pi: X \rightarrow B$ be a morphism satisfying Hypothesis 2.1, and let $f: C \rightarrow X$ be a stable map from an irreducible nodal curve $C$ of genus $g$ to $X$ with class $f_{*}[C]=\beta$. We say that $f$ is flexible relative to $\pi$ if the map $\varphi: \bar{M}_{g, 0}(X, \beta) \rightarrow \bar{M}_{g, 0}(B, d)$ is dominant at the point $[f] \in \bar{M}_{g, 0}(X, \beta)$; that is, if any neighborhood of $[f]$ in $\bar{M}_{g, 0}(X, \beta)$ dominates a neighborhood of $[\pi f]$ in $\bar{M}_{g, 0}(B, d)$.

Now, it is a classical fact that the variety $\bar{M}_{g, 0}(B, d)$ has a unique irreducible component whose general member corresponds to the map $f: C \rightarrow B$ with $C$ a smooth curve (see for example [C] and $[\mathrm{H}]$, and [F, Prop 1.5] for a modern treatment). Since the map $\varphi: \bar{M}_{g, 0}(X, \beta) \rightarrow \bar{M}_{g, 0}(B, d)$ is proper, it follows that if $\pi: X \rightarrow B$ admits a flexible curve, then $\varphi$ will be surjective onto this component. Moreover, this component contains points $[f]$ corresponding to maps $f: C \rightarrow B$ with the property that every irreducible component of $C$ on which $f$ is nonconstant maps isomorphically via $f$ to $B$. (For example, we could simply start with $d$ disjoint copies $C_{1}, \ldots, C_{d}$ of $B$ (with $f$ mapping each isomorphically to $B$ ) and identify $d+g-1$ pairs of points on the $C_{i}$, each pair lying over the same point of $B$. It is easy to check that such a morphism can be smoothed.)

Proposition 2.4. If $\pi: X \rightarrow B$ is a morphism satisfying Hypothesis 2.1 and $f: C \rightarrow X$, a flexible stable map, then $\pi$ has a section.

Our goal in what follows, accordingly, will be to construct a flexible curve $f$ : $C \rightarrow X$ for an arbitrary $\pi: X \rightarrow B$ satisfying Hypothesis 2.1

2.1. The first construction. To manufacture our flexible curve, we apply two basic constructions, which we describe here. (These constructions, especially the first, are pretty standard; see for example section II.7 of [K].) We start with a basic lemma:

Lemma 2.5. Let $C$ be a smooth curve and $E$ any vector bundle on $C$; let $n$ be any positive integer. Let $p_{1}, \ldots, p_{N} \in C$ be general points and $\xi_{i} \subset E_{p_{i}}$ a general onedimensional subspace of the fiber of $E$ at $p_{i}$; let $E^{\prime}$ be the sheaf of rational sections of $E$ having at most a simple pole at $p_{i}$ in the direction $\xi_{i}$ and regular elsewhere. For $N$ sufficiently large we will have

$$
H^{1}\left(C, E^{\prime}\left(-q_{1}-\cdots-q_{n}\right)\right)=0
$$

for any $n$ points $q_{1}, \ldots, q_{n} \in C$.

Proof. To start with, we will prove simply that $H^{1}\left(C, E^{\prime}\right)=0$. Since this is an open condition, it will suffice to exhibit a particular choice of points $p_{i}$ and subspaces $\xi_{i}$ that works. Denoting the rank of $E$ by $r$, we take $N=m r$ divisible by $r$ and choose $m$ points $t_{1}, \ldots, t_{m} \in C$. We then specialize to the case

$$
\begin{array}{rr}
p_{1}=\cdots=p_{r}=t_{1} ; & \xi_{1}, \ldots, \xi_{r} \text { spanning } E_{t_{1}}, \\
p_{r+1}=\cdots=p_{2 r}=t_{2} ; & \xi_{r+1}, \ldots, \xi_{2 r} \text { spanning } E_{t_{2}},
\end{array}
$$

and so on. In this case we have $E^{\prime}=E\left(t_{1}+\cdots+t_{m}\right)$, which we know has vanishing higher cohomology for sufficiently large $m$.

Given this, the statement of the lemma follows: to begin with, choose any $g+n$ points $r_{1}, \ldots, r_{g+n} \in C$. Applying the argument thus far to the bundle $E\left(-r_{1}-\cdots-r_{g+n}\right)$, we find that for $N$ sufficiently large we will have

$$
H^{1}\left(C, E^{\prime}\left(-r_{1}-\cdots-r_{g+n}\right)\right)=0 \text {. }
$$


But now for any points $q_{1}, \ldots, q_{n} \in C$ we have

$$
q_{1}+\cdots+q_{n}=r_{1}+\cdots+r_{g+n}-D
$$

for some effective divisor $D$ on $C$. It follows then that

$$
\begin{aligned}
h^{1}\left(C, E^{\prime}\left(-q_{1}-\cdots-q_{n}\right)\right) & =h^{1}\left(C, E^{\prime}\left(-r_{1}-\cdots-r_{g+n}\right)(D)\right) \\
& \leq h^{1}\left(C, E^{\prime}\left(-r_{1}-\cdots-r_{g+n}\right)\right) \\
& =0 .
\end{aligned}
$$

It is well known that given an embedded curve $D \subset X$ which is at-worst-nodal and given an irreducible component $C \subset D$ which intersects $\overline{D-C}$ in the nodes $p_{1}, \ldots, p_{\delta}$, the restriction $\left.N_{D / X}\right|_{C}$ (as a sheaf) consists of rational sections of $N_{C / X}$ with at worst simple poles at each $p_{i}$ whose normal direction is determined by the other branch of $D$ through $p_{i}$. We will need a version of this result when the embedded curves are replaced by stable maps to $X$.

For an embedded LCI curve $C \subset X$, the space of first-order deformations and the obstruction group are given by $H^{0}\left(C, N_{C / X}\right)$ and $H^{1}\left(C, N_{C / X}\right)$, respectively. Suppose given an unmarked stable map $f: C \rightarrow X$. By [BF] and [B], the space of first-order deformations of the stable map and the obstruction group are given by the hypercohomology groups

$$
\begin{aligned}
& \operatorname{Def}(f)=\mathbb{H}^{1}\left(C, \mathbb{R} \operatorname{Hom}_{\mathcal{O}_{C}}\left(\Omega_{f}, \mathcal{O}_{C}\right)\right), \\
& \operatorname{Obs}(f)=\mathbb{H}^{2}\left(C, \mathbb{R} \operatorname{Hom}_{\mathcal{O}_{C}}\left(\Omega_{f}, \mathcal{O}_{C}\right)\right),
\end{aligned}
$$

where $\Omega_{f}$ is the complex

$$
\begin{array}{cc}
-1 & 0 \\
f^{*} \Omega_{X} \stackrel{d f^{\dagger}}{\longrightarrow} \Omega_{C} .
\end{array}
$$

We will only need to use the deformation theory of stable maps when the stable map satisfies the additional hypothesis that the unramified locus $U \subset C$ contains all nodes of $C$ (in particular there are no irreducible components on which $f$ is constant). In this case $\mathbb{R} \operatorname{Hom}_{\mathcal{O}_{C}}\left(\Omega_{f}, \mathcal{O}_{C}\right)$ is quasi-isomorphic to a complex $\mathcal{N}_{f}[-1]$ where $\mathcal{N}_{f}$ is a coherent sheaf, the normal sheaf of $f$, whose restriction to $U$ is isomorphic to the dual of the kernel of $f^{*} \Omega_{X} \rightarrow \Omega_{C}$.

Suppose $\left(f: C \rightarrow X,\left(p_{1}, \ldots, p_{\delta}\right)\right)$ and $\left(f^{\prime}: C^{\prime} \rightarrow X,\left(p_{1}^{\prime}, \ldots, p_{\delta}^{\prime}\right)\right)$ are stable maps such that

(1) the unramified locus of $f$ (resp. $f^{\prime}$ ) contains all nodes of $f$ as well as all marked points,

(2) for every $i=1, \ldots, \delta, f\left(p_{i}\right)=f^{\prime}\left(p_{i}^{\prime}\right)$, and

(3) for every $i=1, \ldots, \delta$, the tangent lines $d f\left(T_{C, p_{i}}\right), d f^{\prime}\left(T_{C^{\prime}, p_{i}^{\prime}}\right) \subset T_{X, f\left(p_{i}\right)}$ are distinct.

By [BM Theorem 3.6], there is a unique stable map $F: D \rightarrow X$ where $D$ is the connected sum of $C$ and $C^{\prime}$ with $p_{i}$ identified to $p_{i}^{\prime}$, and such that $\left.F\right|_{C}=f$, $\left.F\right|_{C^{\prime}}=f^{\prime}$. Condition (3) above ensures that the points $p_{i}$ are in the unramified locus of $F$. 
Lemma 2.6. Let $X$ be a smooth projective variety and let $(f: C \rightarrow X$, $\left.\left(p_{1}, \ldots, p_{\delta}\right)\right)$ and let $\left(f^{\prime}: C \rightarrow X,\left(p_{1}^{\prime}, \ldots, p_{\delta}^{\prime}\right)\right)$ be as above. Let $(F: D \rightarrow X)$ be the stable map obtained by gluing each $p_{i}$ to $p_{i}^{\prime}$. The normal sheaf of the map $\mathcal{N}_{F}$ is locally free near each of the nodes $p_{i}$ of $D$ and we have an inclusion of sheaves

$$
\left.0 \rightarrow \mathcal{N}_{f} \rightarrow \mathcal{N}_{F}\right|_{C}
$$

identifying $\left.\mathcal{N}_{F}\right|_{C}$ with the sheaf of rational sections of $\mathcal{N}_{f}$ having at most a simple pole at each $p_{i}$ in the normal direction determined by $d f^{\prime}\left(T_{p_{i}^{\prime}} C^{\prime}\right)$. Moreover, if $(\tilde{F}: \tilde{D} \rightarrow X)$ is a first-order deformation of $F$ corresponding to a global section $\sigma \in H^{0}\left(D, \mathcal{N}_{F}\right)$, then $\tilde{D}$ smooths the node of $D$ at $p_{i}$ if and only if the restriction $\left.\sigma\right|_{U}$ of $\sigma$ to a neighborhood $U$ of $p_{i}$ in $C$ is not in the image of $\mathcal{N}_{f}$.

As this lemma concerns only the local behavior near the nodes, it follows immediately from the analogous statement for embedded curves.

Hypothesis 2.7. Suppose $\pi: X \rightarrow B$ is a morphism satisfying our basic Hypothesis 2.1. An unmarked stable map $f: C \rightarrow X$ satisfies our second hypotheses if $C$ is smooth and $\mu:=\pi \circ f$ is surjective.

Suppose $f: C \rightarrow X$ satisfies our second hypotheses and $C$ has genus $g$. For a general point $p \in C$, let $X_{p}=\pi^{-1}(\pi(f(p)))$ be the fiber of $\pi$ through $f(p)$. By hypothesis, $X_{p}$ is a smooth, rationally connected variety, so that we can find a stable map $\left(f^{\prime}: C^{\prime} \subset X_{p}\right)$ from a smooth rational curve and a point $p^{\prime} \rightarrow C^{\prime}$ (at which $f^{\prime}$ is unramified) such that $f(p)=f^{\prime}\left(p^{\prime}\right)$, such that the image of $d f^{\prime}\left(T_{p^{\prime}} C^{\prime}\right)$ in $T_{f(p)} X / d f\left(T_{p} C\right)=\left(\mathcal{N}_{C^{\prime} / X}\right)_{p}$ is arbitrary, and such that $\left(f^{\prime}\right)^{*} T X_{p}$ is an ample bundle on $C^{\prime}$.

Choose a large number of general points $p_{1}, \ldots, p_{\delta} \in C$, and for each $i$ let $f_{i}^{\prime}: C_{i}^{\prime} \subset X_{p_{i}}$ be a stable map as in the last paragraph. Let $F: D \rightarrow X$ denote the stable map obtained by gluing each $C_{i}^{\prime}$ to $C$ by identifying $p_{i}$ and $p_{i}^{\prime}$. Combining the preceding two lemmas, we see that for $\delta$ sufficiently large, the normal sheaf $\mathcal{N}_{F}$ will be generated by its global sections; in particular, by Lemma 2.6 there will be a smooth deformation $\tilde{f}: \tilde{C} \rightarrow X$ of $F$ (i.e., a general stable map which is a member of a 1-parameter family of stable maps specializing to $F$ ). Moreover, for any given $n$ we can choose the number $\delta$ large enough to ensure that

$$
H^{1}\left(C,\left.\mathcal{N}_{F}\right|_{C}\left(-r_{1}-\cdots-r_{g+n}\right)\right)=0
$$

for some $g+n$ points $r_{1}, \ldots, r_{g+n} \in C$; it follows that $H^{1}\left(\tilde{C}, \mathcal{N}_{\tilde{f}}\left(-r_{1}-\cdots-r_{g+n}\right)\right)=$ 0 for some $r_{1}, \ldots, r_{g+n} \in \tilde{C}$ and hence that

$$
H^{1}\left(\tilde{C}, \mathcal{N}_{\tilde{f}}\left(-q_{1}-\cdots-q_{n}\right)\right)=0
$$

for any $n$ points on $\tilde{C}$.

The process of taking a stable map $f: C \rightarrow X$ which satisfies our second hypotheses, attaching rational curves in fibers and smoothing as above to get a new stable map $\tilde{f}: \tilde{C} \rightarrow X$ with smooth domain, is our first construction. It has the properties that:

(1) $\tilde{f}: \tilde{C} \rightarrow X$ also satisfies our second hypotheses,

(2) the genus $g$ of the new curve $\tilde{C}$ is the same as the genus of the curve $C$ we started with,

(3) the degree $d$ of $\tilde{\mu}=\pi \circ \tilde{f}: \tilde{C} \rightarrow B$ is the same as the degree of $\mu=\pi \circ f$ : $C \rightarrow B$, 
(4) the branch divisor of $\tilde{\mu}$ is a small deformation of the branch divisor of $\mu$, and again,

(5) for any $n$ points $q_{1}, \ldots, q_{n} \in \tilde{C}$ we have $H^{1}\left(\tilde{C}, \mathcal{N}_{\tilde{f}}\left(-q_{1}-\cdots-q_{n}\right)\right)=0$.

Here is one application of this construction. Suppose we have a stable map $f: C \subset X$ satisfying our second hypotheses such that the projection $\mu$ is simply branched - that is, the branch divisor of $\mu$ consists of $2 d+2 g-2$ distinct points in $B$ - and such that each ramification point $p \in C$ of $\mu$ maps to a smooth point of the fiber $X_{p}$. Applying our first construction with $n=2 d+2 g-2$, we arrive at another stable map $\tilde{f}: \tilde{C} \rightarrow X$ satisfying our hypotheses which is again simply branched over $B$, with all ramification points mapping to smooth points of fibers of $\pi$. But now the condition that $H^{1}\left(\tilde{C}, \mathcal{N}_{\tilde{f}}\left(-q_{1}-\cdots-q_{n}\right)\right)=0$ applied to the $n=2 d+2 g-2$ ramification points of the map $\tilde{\mu}=\pi \circ \tilde{f}$ says that if we pick a normal vector $v_{i} \in\left(\mathcal{N}_{\tilde{f}}\right)_{p_{i}}$ at each ramification point $p_{i}$ of $\tilde{\mu}$, we can find a global section of the normal sheaf $\mathcal{N}_{\tilde{f}}$ with value $v_{i}$ at $p_{i}$. Moreover, since ramification occurs at smooth points of fibers of $\pi$, for any tangent vectors $w_{i}$ to $B$ at the image points $\tilde{\mu}\left(p_{i}\right)$ we can find tangent vectors $v_{i} \in\left(\mathcal{N}_{\tilde{f}}\right)_{p_{i}}$ with $d \pi\left(v_{i}\right)=w_{i}$. It follows that as we deform the stable map $\tilde{f}: \tilde{C} \rightarrow X$, the branch points of $\tilde{\mu}$ move independently. A general deformation of $\tilde{f}$ thus yields a general deformation of $\tilde{\mu}$ - in other words, the stable map $\tilde{f}$ is flexible. We thus make the

Definition 2.8. Let $\pi: X \rightarrow B$ be as in Hypothesis 2.1 and let $f: C \subset X$ be a stable map as in Hypothesis 2.7 such that the projection $\mu=\pi \circ f$ is simply branched. If each ramification point $p \in C$ of $\mu$ maps to a smooth point of the fiber $X_{p}$ containing it, we will say the stable map $f: C \rightarrow X$ is pre-flexible.

In these terms, we have established the

Lemma 2.9. Let $\pi: X \rightarrow B$ be as in Hypothesis 2.1. If $X$ admits a pre-flexible stable map, the map $\pi$ has a section.

2.2. The second construction. Our second construction is a very minor modification of the first. Given a family $\pi: X \rightarrow B$ as in Hypothesis 2.1 and a stable map $f: C \rightarrow X$ satisfying Hypothesis [2.7, we pick a general fiber $X_{b}$ of $\pi$ and two points $p, q \in C$ such that $f(p), f(q) \in X_{b}$. We then pick a stable map $f_{0}^{\prime}: C_{0}^{\prime} \rightarrow X_{b}$ with smooth, rational domain and two points $p^{\prime}, q^{\prime} \in C_{0}^{\prime}$ such that $f_{0}^{\prime}\left(p^{\prime}\right)=f(p)$ and $f_{0}^{\prime}\left(q^{\prime}\right)=f(q)$ such that $\left(f_{0}^{\prime}\right)^{*} T X_{b}$ is an ample bundle.

We also pick a large number $N$ of other general points $p_{i} \in C$ and stable maps $f_{i}^{\prime}: C_{i}^{\prime} \rightarrow X_{p_{i}}$ in the corresponding fibers and points $p_{i}^{\prime} \in C_{i}^{\prime}$ such that $f_{i}^{\prime}\left(p_{i}^{\prime}\right)=$ $f\left(p_{i}\right)$, such that $\left(f_{i}^{\prime}\right)^{*} T X_{p_{i}}$ are ample and such that $d f_{i}^{\prime}\left(T_{p_{i}^{\prime}} C_{i}^{\prime}\right)$ is a general line in $T_{f\left(p_{i}\right)} X_{p_{i}}$ (just as in the first construction). Finally, we let $F: D \rightarrow X$ be the stable map obtained by gluing $C$ and $C_{0}^{\prime}, C_{1}^{\prime}, \ldots, C_{N}^{\prime}$ by identifying $p$ with $p^{\prime}, q$ with $q^{\prime}$ and for each $i$ identifying $p_{i}$ with $p_{i}^{\prime}$. As in the first construction, for $N$ large enough, there will be deformations of $F: D \rightarrow X$ with smooth domain, and we choose $\tilde{f}: \tilde{C} \rightarrow X$ a general smooth deformation of $D$ (i.e., a general stable map which is a member of a 1-parameter family of stable maps specializing to $F$ ). This process, starting with the stable map $f: C \rightarrow X$ and arriving at the new stable map $\tilde{f}: \tilde{C} \rightarrow X$, is our second construction. It has the properties that

(1) $\tilde{f}: \tilde{C} \rightarrow X$ satisfies Hypothesis 2.7

(2) the degree $d$ of $\tilde{\mu}:=\pi \circ \tilde{f}$ is the same as the degree of $\mu:=\pi \circ f$, 
(3) the genus of the new curve $\tilde{C}$ is one greater than the genus of the curve $C$ we started with,

(4) for any $n$ points $q_{1}, \ldots, q_{n} \in \tilde{C}$ we have $H^{1}\left(\tilde{C}, \mathcal{N}_{\tilde{f}}\left(-q_{1}-\cdots-q_{n}\right)\right)=0$, and

(5) the branch divisor of $\mu$ has two new points: it consists of a small deformation of the branch divisor of $\mu$, together with a pair of simple branch points $b^{\prime}, b^{\prime \prime} \in B$ near $b$, each having as monodromy the transposition exchanging the sheets of $\tilde{C}$ near $p$ and $q$.

In effect, we have simply introduced two new simple branch points to the cover $C \rightarrow B$, with assigned (though necessarily equal) monodromy. Note that we can apply this construction repeatedly, to introduce any number of (pairs of) additional branch points with assigned (simple) monodromy; or we could carry out a more general construction with a number of curves $C_{0}$.

\section{Proof OF THE MAIN THEOREM}

3.1. The proof in case $B=\mathbb{P}^{1}$. We are now more than amply equipped to prove the theorem. We start with a morphism $\pi: X \rightarrow B$ as in Hypothesis 2.1 To begin with, by hypothesis $X$ is projective; embed in a projective space and take the intersection with $\operatorname{dim}(X)-1$ general hyperplanes to arrive at a smooth curve $C \subset X$. The inclusion $f: C \hookrightarrow X$ satisfies Hypothesis 2.7 and is the stable map we start with.

What do $C$ and the associated map $\mu: C \hookrightarrow X \rightarrow B$ look like? To answer this, start with the simplest case: suppose that the fibers $X_{b}$ of $\pi$ do not have multiple components, or in other words that the singular locus $\pi_{\text {sing }}$ of the map $\pi$ has codimension 2 in $X$. In this case we are done: $C$ misses $\pi_{\text {sing }}$ altogether, so that all ramification of $\mu: C \rightarrow B$ occurs at smooth points of fibers; and simple dimension counts show we can choose $C$ so that the branching of $\mu$ is simple. In other words, $f: C \hookrightarrow X$ is pre-flexible already.

The problems start if $\pi$ has multiple components of fibers. If $Z \subset X_{b}$ is such a component, then each point $p \in C \cap Z$ will be a ramification point of $\mu$, and no deformation of $C$ will move the corresponding branch point $\pi(p) \in B$. The curve $C$ cannot be flexible. And of course it is worse if $\pi$ has a multiple (that is, everywhere-nonreduced) fiber: in that case $\pi$ cannot possibly have a section (a posteriori we will see this cannot occur).

To keep track of such points, let $M \subset B$ be the locus of points such that the fiber $X_{b}$ has a multiple component. Outside of $M$, the map $\mu: C \rightarrow B$ is simply branched, and all ramification occurs at smooth points of fibers of $\pi$.

Now here is what we are going to do. First, pick a base point $p_{0} \in B$, and draw a cut system: that is, a collection of real arcs joining $p_{0}$ to the branch points $M \cup N$ of $\mu$, disjoint except at $p_{0}$. The inverse image in $C$ of the complement $U$ of these arcs is simply $d$ disjoint copies of $U$; call the set of sheets $\Gamma$ (or, if you prefer, label them with the integers 1 through $d$ ). Now, for each point $b \in M$, denote the monodromy around the point $b$ by $\sigma_{b}$, and express this permutation of $\Gamma$ as a product of transpositions:

$$
\sigma_{b}=\tau_{b, 1} \tau_{b, 2} \cdots \tau_{b, k_{b}}
$$

so that in other words

$$
\tau_{b, k_{b}} \cdots \tau_{b, 2} \tau_{b, 1} \sigma_{b}=I
$$

is the identity. For future reference, let $k=\sum k_{b}$. We will proceed in three stages. 


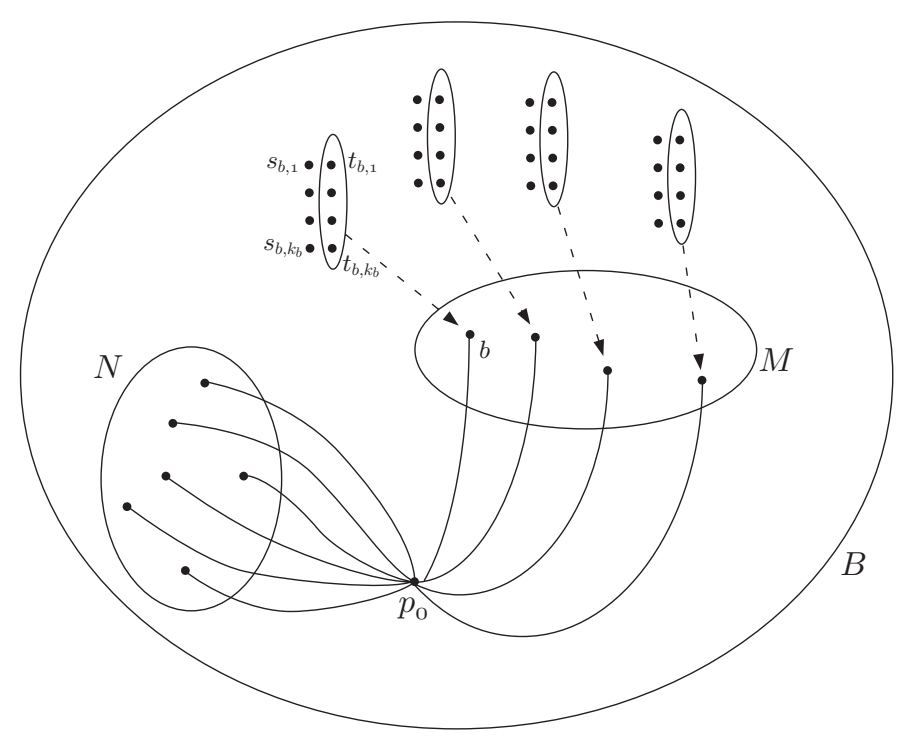

FIGURE 1. Specializing branch points

Stage 1: We use our second construction to produce a new stable map $\tilde{f}: \tilde{C} \rightarrow X$ such that $\tilde{f}$ is unramified at $\tilde{f}^{-1}(M)$ and such that in a neighborhood of each point $b \in M$ we have $k_{b}$ new pairs of simple branch points of $\tilde{\mu}=\pi \circ \tilde{f}$, say $s_{b, i}, t_{b, i} \in B$, with the monodromy around $s_{b, i}$ and $t_{b, i}$ equal to $\tau_{b, i}$. Note that $\tilde{C}$ will have genus $g(C)+k$ and that the branch divisor of the projection $\tilde{\mu}$ will be the union of $M$, of a small deformation $\tilde{N}$ of $N$, and of all the points $s_{b, i}$ and $t_{b, i}$. In particular we can find disjoint discs $\Delta_{b} \subset B$, with $\Delta_{b}$ containing the points $b$ and $t_{b, 1}, t_{b, 2}, \ldots, t_{b, k_{b}}$, so that the monodromy around the boundary $\partial \Delta_{b}$ of $\Delta_{b}$ is trivial.

Now, for any fixed integer $n$ this construction can be carried out so that the stable map $\tilde{f}$ has the property that $H^{1}\left(\tilde{C}, \mathcal{N}_{\tilde{f}}\left(-q_{1}-\cdots-q_{n}\right)\right)=0$ for any $n$ points $q_{i} \in \tilde{C}$. Here we want to choose

$$
n=\# N+2 k
$$

so that there are global sections of the normal sheaf $\mathcal{N}_{\tilde{f}}$ with arbitrarily assigned values on the ramification points of $\tilde{C}$ over $N$ and the points $s_{b, i}$ and $t_{b, i}$. This means in particular that we can deform the stable map $\tilde{f}: \tilde{C} \rightarrow X$ so as to deform the branch points of $\tilde{\mu}$ outside of $M$ independently. What we will do, then, is

Stage 2: We will vary $\tilde{f}: \tilde{C} \rightarrow X$ so as to keep all the branch points $b \in N$ and all the points $s_{b, i}$ fixed; and for each $b \in M$ specialize all the branch points $t_{b, i}$ to $b$ within the disc $\Delta_{b}$.

This is illustrated in Figure 1. To say this more precisely, let $\beta \in N_{1}(X)$ be the class of the stable map $\tilde{f}: \tilde{C} \rightarrow X$, and consider the maps

$$
\bar{M}_{g^{\prime}, 0}(X, \beta) \longrightarrow \bar{M}_{g^{\prime}, 0}(B, d) \longrightarrow B_{2 d+2 g^{\prime}-2}
$$

with the second map assigning to a stable map $C \rightarrow B$ its branch divisor (we only need the branch morphism as a set map, but in fact it is a regular morphism [ $\mathrm{FaP}]$ ). 
What we are saying is, starting at the branch divisor

$$
D_{1}=\tilde{N}+\sum s_{b, i}+\sum t_{b, i}+\sum_{b \in M} k_{b} \cdot b
$$

of the map $\tilde{\mu}$, draw an analytic arc $\gamma=\left\{D_{\lambda}\right\}$ in the subvariety

$$
\Phi=\tilde{N}+\sum s_{b, i}+\sum_{b \in M} k_{b} \cdot b+\sum\left(\Delta_{b}\right)_{k_{b}} \subset B_{2 d+2 g^{\prime}-2}
$$

tending to the point

$$
D_{0}=\tilde{N}+\sum s_{b, i}+2 \sum_{b \in M} k_{b} \cdot b .
$$

Since the image of the composition

$$
\bar{M}_{g^{\prime}, 0}(X, \beta) \longrightarrow B_{2 d+2 g^{\prime}-2}
$$

contains $\Phi$, we can find an $\operatorname{arc} \delta=\left\{f_{\nu}\right\}$ in $\bar{M}_{g^{\prime}, 0}(X, \beta)$ that maps onto $\gamma$, with $f_{1}$ the inclusion $\tilde{C} \hookrightarrow X$.

Stage 3: Let $f_{0}: C_{0} \rightarrow X$ be the limit, in $\bar{M}_{g^{\prime}, 0}(X, \beta)$, of the family of curves constructed in Stage 2; that is, the point of the arc $\delta$ over $D_{0} \in \Phi \subset B_{2 d+2 g^{\prime}-2}$. Let $A \rightarrow C_{0}$ be the normalization of any irreducible component of $C_{0}$ on which the composition $\pi f_{0}$ is nonconstant (that is, whose image is not contained in a fiber), and let $f_{A}: A \rightarrow X$ be the stable map where $f_{A}$ is the restriction of $f_{0}$ to $A$. In particular, $f_{A}: A \rightarrow X$ is a stable map satisfying Hypothesis 2.7 .

By construction, the composition $\mu_{A}:=\pi \circ f_{A}$ is unramified over a neighborhood of $M$ : the monodromy around the boundary $\partial \Delta_{b}$ of each disc $\Delta_{b}$ is trivial, and it can be branched over at most one point $b$ inside $\Delta_{b}$, so it cannot be branched at all over $\Delta_{b}$. Indeed, it is (at most) simply branched over each point of $N$ and each point $s_{b, i}$, and unramified elsewhere. Moreover, since we can carry out the specialization of $\tilde{f}: \tilde{C} \rightarrow X$ above with the entire fiber of $\tilde{\mu}$ over the points of $N$ and the $s_{b, i}$ fixed, the ramification of $\mu_{A}$ over these points will occur at smooth points of the corresponding fibers of $\pi$. In other words, the map $f_{A}: A \rightarrow X$ is pre-flexible, and we are done.

3.2. The proof for arbitrary curves $B$. As we indicated at the outset, there are two straightforward ways of extending this result to the case of arbitrary curves $B$.

For one thing, virtually all of the argument we have made goes over without change to the case of base curves $B$ of any genus $h$. The one exception to this is the statement that the space $\bar{M}_{g, 0}(B, d)$ of stable maps $f: C \rightarrow B$ of degree $d$ from curves $C$ of genus $g$ to $B$ has a unique irreducible component whose general member corresponds to a flat map $f: C \rightarrow B$. This is false in general-consider for example the case $g=d(h-1)+1$ of unramified covers. It is true, however, if we restrict ourselves to the case $g \gg h, d$ (that is, we have a large number of branch points) and look only at covers whose monodromy is the full symmetric group $S_{d}$ GHS Theorem 1]. Given this fact and observing that our second construction allows us to increase the number of branch points of our covers $C \rightarrow B$ arbitrarily, the theorem can be proved for general $B$ just as it is proved above for $B \cong \mathbb{P}^{1}$.

Alternatively, Johan de Jong showed us a simple way to deduce the theorem for general $B$ from the case $B \cong \mathbb{P}^{1}$ alone. We argue as follows: given a map $\pi: X \rightarrow B$ with rationally connected general fiber, we choose any map $g: B \rightarrow \mathbb{P}^{1}$ 
expressing $B$ as a branched cover of $\mathbb{P}^{1}$. We can then form the "norm" of $X$ : this is the (birational isomorphism class of) variety $Y \rightarrow \mathbb{P}^{1}$ whose fiber over a general point $p \in \mathbb{P}^{1}$ is the product

$$
Y_{p}=\prod_{q \in g^{-1}(p)} X_{q} .
$$

Since the product of rationally connected varieties is again rationally connected, it follows from the $\mathbb{P}^{1}$ case of the theorem that $Y \rightarrow \mathbb{P}^{1}$ has a rational section, and hence so does $\pi$.

\section{ACKNOWLEDGMENTS}

We would like to thank Johan de Jong, János Kollár and Barry Mazur for many conversations, which were of tremendous help to us. We would also like to thank Olivier Debarre, Vyacheslav Shokurov and the referee for useful comments on preliminary versions of this work.

\section{REFERENCES}

[B] K. Behrend, Gromov-Witten invariants in algebraic geometry, Invent. math. 127 (1997), 601-617. MR 98i:14015

[BF] K. Behrend, B. Fantechi, The intrinsic normal cone, Invent. math. 128 (1998), 45-88. MR 98e:14022

[BM] K. Behrend, Y. Manin, Stacks of stable maps and Gromov-Witten invariants, Duke Math J. 85, 1-60. MR 98i:14014

[Ca] F. Campana, Connexité rationnelle des variétés de Fano, Ann. Sc. E. N.S. 25 (1992), 539-545. MR 93k:14050

[C] A. Clebsch, Zur Theorie der Riemann'schen Flachen, Math Ann. 6 (1872), 216-230 Springer-Verlag, Berlin, 1996.

[FaP] B. Fantechi, R. Pandharipande, Stable maps and branch divisors, Compositio Math. 130 (2002), 345-364.

[F] W. Fulton, Hurwitz schemes and moduli of curves, Annals of Math. 90 (1969), 542-575. MR 41:5375

[FP] W. Fulton, R. Pandharipande, Notes on stable maps and quantum cohomology, 45-96, in Algebraic geometry - Santa Cruz 1995, AMS, 1995. MR 98m:14025

[GHS] T. Graber, J. Harris, J. Starr, A note on Hurwitz schemes of covers of a positive genus curve, preprint alg-geom/0205056.

[H] A. Hurwitz, Ueber Riemann'sche Flächen mit gegebenen Verzweigungspunkten, Math. Ann. 39 (1891) 1-61.

[K] J. Kollár, Rational Curves on Algebraic Varieties, Ergebnisse der Math. 32, SpringerVerlag, Berlin, 1996. MR 98c:14001

[KMM] J. Kollár, Y. Miyaoka, S. Mori, Rationally Connected Varieties, J. Alg. Geom. 1 (1992), 429-448. MR 93i:14014

Department of Mathematics, Harvard University, 1 Oxford Street, Cambridge, MASSACHUSETTS 02138

E-mail address: graber@math.harvard.edu

Department of Mathematics, Harvard University, 1 Oxford Street, Cambridge, MASSACHUSETTS 02138

E-mail address: harris@math.harvard.edu

Department of Mathmatics, Massachusetts Institute of technology, Cambridge, MASSACHUSETtS 02139

E-mail address: jstarr@math.mit.edu 